\title{
ANTIMICROBIALS FOR FOOD PRESERVATION DELIVERED IN NANOSIZED MATRICES
}

\author{
Mihaela Leonida* and Alice Benzecry \\ School of Natural Sciences, Fairleigh Dickinson University, Teaneck, NJ 07666, \\ USA, email: mleonida@fdu.edu
}

\begin{abstract}
Food safety is a major concern in the food industry and bacterial contamination of products is a high concern for human health. Hops and the components extracted from them are well known antibacterial agents used in beers and as food preservatives, on their own or together with other antimicrobial agents, and as antioxidants. However, their very low bioavailability, bitter taste, and susceptibility to oxidative decomposition have limited their applications. The use of proteins and peptides as antimicrobials in the food industry has expanded but problems are encountered due to their susceptibility to proteolytic degradation, low solubility, and, physicochemical instability. We propose nanosized chitosan, an inexpensive, readily available biopolymer with a broad spectrum of antibacterial activity, as carrier for lupulone (L) and xanthohumol (X), two components of hops, and for nisin (N), a lantibiotic used as food preservative. Chitosan nanoparticles (CNP) and chitosan-based nanocomposites encapsulating lupulone (CNPL), xanthohumol (CNPX), and nisin (CNPN) were prepared by ionotropic gelation. The nanoparticles obtained were characterized by FTIR, colloidal titration, encapsulation efficiency, loading capacity, and antimicrobial activity. The kinetics of the release of $\mathrm{L} / \mathrm{X} / \mathrm{N}$ from composites was studied in vitro. All the nanoparticles were active against several Gram positive and Gram-negative microbial species important for food preservation. Although hops and nisin are known mainly for their activity against Gram positive bacteria, synergistic interactions with chitosan broaden their activity against Gram negative pathogens. All nanocomposites maintained antimicrobial effect over an extended period of time.
\end{abstract}

Keywords: lupulone, xanthohumol, nisin, food preservation, nanochitosan, encapsulation efficiency, release kinetics, antimicrobials.

\section{Introduction}

The food industry is very interested in antibacterial species active against food pathogens (both Gram positive and Gram negative), yeasts, and spores. They need to be active against a broad spectrum of species and stable. Filmogenic antimicrobials are of special interest.

As an inexpensive, biocompatible polymer, chitosan (Figure 1) has been used in the medical field (orthopedics, hypercholesterolemia) and in pharmaceuticals (as drug delivery vehicle) [1,2]. Chitosan nanoparticles were reported to have strong biological activities and to be good agents used to increase bioavailability of poorly soluble drugs. This type of material is good as food preservative due to its filmogenic properties combined with its inhibitory activity against bacteria (both Gram positive and Gram negative), spores, and yeasts $[3,4]$. It acts by increasing the permeability of cell membranes due to favorable interaction between the protonated amine groups of chitosan and the negatively charged cell membranes. This leads to leakage of intracellular constituents. As a chelator, chitosan can remove metals and other essential elements from the cells making its antibacterial effect a rather complex one. 


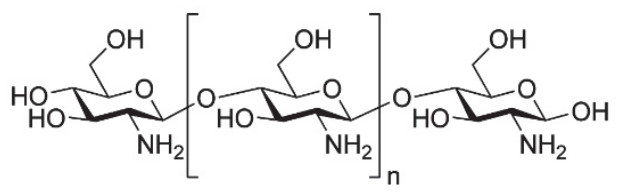

Figure 1. Chitosan

Bacteriocins are toxins with peptidic structures, secreted by bacterial species to protect themselves. They inhibit the growth of similar bacterial species. Nisin $(\mathrm{N})$, a bacteriocin, is obtained by fermentation of dairy or dextrose nutrients in the presence of Lactococcus lactis cultures. It is a polycyclic antibacterial peptide (34 amino acids, Figure 2 ), used as a food preservative since it is active against Gram positive bacteria and against spores [5]. In the food industry, nisin is useful in controlling spoilage due to lactic acid bacteria in alcoholic beverages (wines, beers), salad dressings, bakery products, meats, fish, and in cheeses.

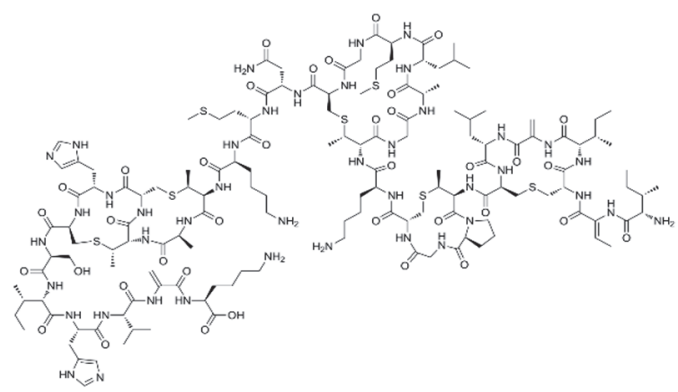

Figure 2. Nisin

Nisin is a suitable food preservative, used in more than fifty countries, because it is nontoxic, safe (food-grade), quickly digested, and it does not give cross-resistance in bacteria (which may lead to antibiotic resistance in subjects being treated for infections) . Nisin is also considered a natural food preservative since it is not the result of any man-conducted chemical synthesis. The main drawback of this agent is its lack of inhibitory activity against Gram negative bacteria, yeasts, and molds, its fast release in lipophilic environments, and its low stability.

Hop constituents (lupulone, xanthohumol, humulone) are known for their antibacterial effect against Gram positive species [6,7].
Their bioavailability is very limited due to their high lipohilicity which makes their release, when used as preservatives in foods, too fast for industrial applications. Lupulone ( $\mathrm{L}$, beta acid, Fig.3a) is one of the bitter acids in hops. It is a mixture of isomers (n-, co-, and ad-lupulone) [8] and it displays the strongest antibacterial effect among hop constituents [9]. Xanthohumol (X, Fig.3b) is the most abundant prenylated flavonoid in the hop plant. It has a broad spectrum of biological activities among which antibacterial [10] and antioxidant [11] are the best known.

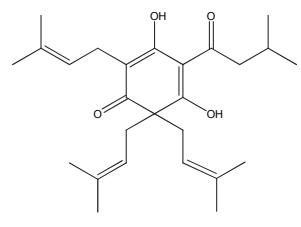

(a)

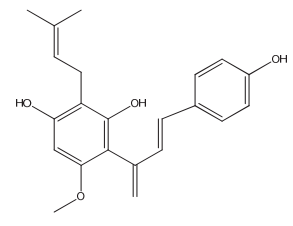

(b)
Figure 3. (a) lupulone; (b) xanthohumol

While having high antimicrobial activity, nisin and hop extracts have limited applications due to fast release (related to high lipophilicity) and poor stability. In the case of hops, their extremely bitter taste limits the number of their applications as preservatives in foods [6] as does their susceptibility to oxidative decomposition. Nanotechnology has offered lately a solution to this type of problems. These shortcomings disappear upon encapsulation in nanosized matrices. More advantages may result if encapsulation is done in structures presenting bioactive properties (like chitosan). The purpose of the present study was to prepare and characterize nanochitosan matrices in which to encapsulate nisin or hop components (lupulone/xanthohumol) in order to improve their performance as food preservatives. Due to the antibacterial activity reported for both chitosan and the encapsulated species, synergistic effects were also investigated.

\section{Experimental Section}

\subsection{Materials and Microorganisms}

Chitosan (CS) prepared from chitin from shrimp, by deacetylation and partial depolymerization, was purchased from Millipore-Sigma (practical grade - CS-PG, 
DDA $\geq 75 \%$, molar weight $190-235 \mathrm{kDa}$ ). The other chemicals were purchased from Millipore-Sigma, USA. They were reagent grade and used without further purification.

Nisin from Lactococcus lactis $(2.5 \%$ nisin content, balanced with sodium chloride and denatured milk solids, MW 3,354 g/mol), was obtained from Millipore-Sigma, USA. The lupulone (purity 98\%) and xanthohumol (purity 100\%) were donated by the SS Steiner Company. The purity was determined in the SS Steiner Laboratories in Yakima, WA, using the HPLC method established at the European Brewery Convention in 2004.

Table 1. List of microbial strains used in the experiments

\begin{tabular}{|c|c|}
\hline Name of organism & $\begin{array}{c}\text { Culture } \\
\text { specification }\end{array}$ \\
\hline $\begin{array}{c}\text { Staphylococcus } \\
\text { aureus }\end{array}$ & ATCC 25923 \\
\hline $\begin{array}{c}\text { Pseudomonas } \\
\text { aeruginosa }\end{array}$ & ATCC 27853 \\
\hline Escherichia coli & ATCC 25922 \\
\hline
\end{tabular}

Microbial cultures were purchased from the American True Culture Collection (ATCC) and are listed in Table 1. The medium used in the experiments was Mueller-Hinton broth (Becton Dickinson BBL 212322).

\subsection{Methods \\ 2.2.1 Preparation of the chitosan nanoparticles and of the nanocomposites}

Chitosan nanoparticles (CNP) were prepared by the ionotropic gelation method [12] adapted by us. A chitosan $0.5 \% \mathrm{w} / \mathrm{v}$ solution was made in $1 \%$ acetic acid (v/v), was ultrasonicated for $30 \mathrm{~min}$, and it was left under magnetic stirring for 24 hours, to complete dissolution. After adjusting the $\mathrm{pH}$ to $\mathrm{pH} 5.5$, sodium tripolyphosphate (TPP) $0.25 \%$ in deionized water was added in a $2: 1$ CS: TPP volume ratio and the mixture was stirred for $1 \mathrm{~h}$. Nanoparticles formed spontaneously as a result of ionic interactions between the positively charged amino groups of CS and the negatively charged groups of TPP. The solutions were centrifuged at $9,000 \mathrm{~g}$ for $45 \mathrm{~min}$, at $20{ }^{\circ} \mathrm{C}$. The supernatant was discarded and the pellets were rinsed with deionized water and left to air dry. Dried pellets were weighed, ground, and stored at $4{ }^{\circ} \mathrm{C}$.

To prepare the chitosan nanocomposites encapsulating $\mathrm{L} / \mathrm{X} /$, respectively, one milliliter of a $8 \mathrm{mg} / \mathrm{mL}$ solution of $\mathrm{L} / \mathrm{X}$ in ethanol was added to $200 \mathrm{~mL}$ solution $0.5 \%$ chitosan in $1 \%$ acetic acid, followed by 30 min ultrasonication. For the nisin containing nanocomposites, $0.1 \mathrm{mg} / \mathrm{mL}$ nisin was added to the chitosan solution, as above, followed by $30 \mathrm{~min}$ ultrasonication. $24 \mathrm{~h}$ stirring ensued, followed by TPP addition, $1 \mathrm{~h}$ stirring, and centrifugation. The supernatants from the centrifugations of nanocomposites were used in the indirect determination of the encapsulated plant extracts/nisin.

\subsubsection{Characterization of the nanoparticles}

For the determination of the $\mathrm{L} / \mathrm{X} / \mathrm{N}$ content of the particles, the volumes of the supernatants from the preparation of the loaded nanoparticles were measured and absorbance values at 342/370/280 nm, respectively, were recorded. Encapsulation efficiency (EE, (1) ) and loading capacity (LC, (2) ) were determined as in [13]:

$$
\begin{gathered}
\% \mathrm{EE}= \\
{\left[\frac{(\text { total amount of } \mathrm{X} / \mathrm{L}-\text { free amount } \mathrm{X} / \mathrm{L})}{\text { total amount } \mathrm{X} / \mathrm{L}}\right] \times 100}
\end{gathered}
$$

$\% \mathrm{LC}=$

$\left[\frac{(\text { total amount X/L }- \text { free amount X/L) }}{\text { mass nanoparticle }}\right] \times 100$

FTIR spectra were taken using the PerkinElmer FTIR Spectrophotometer Spectrum Two, equipped with ATR accessory. Finely ground samples were placed on the ATR crystal and 254 co-added scans at $4 \mathrm{~cm}^{-1}$ resolution were taken on each sample.

The free positively charged amino groups on the nanoparticles were determined by colloidal titration (with potassium poly(vinyl sulfate) (PVSK) in the presence of toluidine blue as indicator) [14]. The number of residual (positively charged) amino groups per gram, calculated as shown in (3), was used to quantitatively evaluate the extent of the 
crosslinking.

Amino groups/g of CNP $=\Delta \mathrm{V} \times \mathrm{M} \times \frac{\mathrm{Na}}{\mathrm{m}}$

where $\Delta \mathrm{V}$ is the net volume of PVSK (L) consumed in the titration, $\mathrm{M}$ is molar concentration of PVSK $(\mathrm{mol} / \mathrm{L}), \mathrm{N}_{\mathrm{a}}$ is Avogadro's number $\left(6.023 \bullet 10^{23}\right.$ molecules/mole), and $m$ is the mass of CNP in the sample $(\mathrm{g})$. The ratio of residual amino groups (RRAG) on the surface of chitosan nanoparticles was calculated using (4):

$\%$ RRAG $=$ $\left[\frac{\text { amino groups/g CNP (after cross-linking) ) }}{\text { (total amino groups/g CS (before cross-linking) }}\right] \times$

\subsubsection{Evaluation of $L / X / N$ release in vitro}

The release profiles for $\mathrm{L} / \mathrm{X} / \mathrm{N}$ from nanoparticles were determined using the sample and separate method [15]. For the kinetics study $0.1 \mathrm{~g}$ of each type of composite was placed in $50 \mathrm{ml}$ of phosphate buffer $(\mathrm{pH}$ 7) to achieve a concentration of $0.002 \mathrm{~g} / \mathrm{ml}$ and the solutions were stirred at room temperature. At specified collection times, 3 $\mathrm{ml}$ samples were withdrawn (and replaced by $3 \mathrm{ml}$ of fresh buffer), filtered, and analyzed for $\mathrm{L} / \mathrm{X} / \mathrm{N}$ content at 342/370/280 $\mathrm{nm}$, respecttively. The instrument used was a DU 640 UV spectrometer and phosphate buffer $\mathrm{pH} 7$ was used as blank. The total mass of released plant extract/nisin $\left(\mathrm{m}_{\mathrm{i}}\right)$ and the percent extract released at time i were calculated according to (5) and (6):

$$
\mathrm{m}_{\mathrm{i}}=\left(\mathrm{c}_{\mathrm{i}} \mathrm{V}+\sum \mathrm{c}_{\mathrm{i}-1} \mathrm{~V}_{\mathrm{s}}\right) \bullet \mathrm{M}
$$

$$
\left[\frac{\mathrm{mi} \text { released }=}{\text { (total amount of } \mathrm{L} / \mathrm{X} \text { in the nanoparticle sample) }}\right] \times
$$

where: $c_{i}$ is the concentration of $L / X / N$ released in solution at time $\mathrm{i}, \mathrm{V}$ is the total volume of solution $(50 \mathrm{ml}), \mathrm{V}_{\mathrm{s}}$ is the volume of a sample solution $(3 \mathrm{ml}), \mathrm{M}$ is the molar weight of the corresponding species $(\mathrm{L} / \mathrm{X} / \mathrm{N})$.

\subsubsection{Antimicrobial assays}

In vitro antimicrobial susceptibility testing was conducted following the Clinical and Laboratory Standards Institute (CLSI) turbidimetric method [16]. To standardize the inoculum density for the susceptibility test, the visual turbidity equivalent to 0.5 McFarland standards containing approximately $1-2 \times 10^{8}$ colony-forming units $(\mathrm{CFU} / \mathrm{ml})$ were used. Broth $(10 \mathrm{ml})$ solutions containing concentrations of $1000 \mu \mathrm{g} / \mathrm{mL}$, $500 \mu \mathrm{g} / \mathrm{mL}, 250 \mu \mathrm{g} / \mathrm{mL}, 125 \mu \mathrm{g} / \mathrm{mL}, 62$ $\mu \mathrm{g} / \mathrm{mL}$ and $31 \mu \mathrm{g} / \mathrm{mL}$ of CS/CNP/CNPL/

CNPX/CNPN were prepared. The $\mathrm{pH}$ of all solutions was adjusted to 5.5 prior to being autoclaved. Each tube was inoculated under aseptic conditions with $0.1 \mathrm{~mL}$ of bacterial standardized solution. Both negative (broth and particles only) and positive (broth with testing microbe only) controls were also prepared. An ethanol control without hops was included in each assay set for hops. All tubes containing bacterial cultures were incubated at $37^{\circ} \mathrm{C}$ for $20-24$ hours (until positive control tubes indicated growth). Subsequently, each tube was examined against the negative and the positive controls for evidence of growth. Each test was done in duplicate, repeated several times and the minimum inhibitory concentration (MIC) values were determined.

\section{RESULTS AND DISCUSSION}

Nanoparticulate materials encapsulating $\mathrm{L} / \mathrm{X} / \mathrm{N}$ were prepared by crosslinking chitosan with sodium tripolyphosphate. The inexpensive, green procedure is based on the electrostatic attraction between protonated amine groups in chitosan and the anionic tripolyphosphate, at room temperature. Table 2 presents the RRAG, EE and LC values for the nanocomposites.

RRAG values are informative when it is necessary to determine surface charges in dilute solutions of polymeric species like the ones discussed herein, and to evaluate crosslinking. Table 2 shows that presence of lupulone in the composites results in a more important decrease in RRAG compared to xanthohumol, and encapsulated nisin affords nanocomposites with the lowest RRAG values. This shows that, while the impact of 
the encapsulation of hydrophobic molecules in nanochitosan is sizable, the disruption generated by the larger amphoteric nisin is more important. All RRAG values recommend these nanoparticles as potential antimicrobial agents. All agents were encapsulated with high efficiency (highest for $\mathrm{X}$ ) while the loading capacity values were low. In loading capacity, hydrophobicity seems to be more important than size of the encapsulated species.

Table 2. Characteristics of the nanoparticles

\begin{tabular}{|c|c|c|c|}
\hline Particle & $\begin{array}{c}\text { RRAG } \\
(\%)\end{array}$ & $\begin{array}{c}\text { EE } \\
(\%)\end{array}$ & $\begin{array}{c}\text { LC } \\
(\%)\end{array}$ \\
\hline CS & 100 & - & - \\
\hline CNP & 79.41 & - & - \\
\hline CNPL & 67.7 & 81.9 & 1.04 \\
\hline CNPX & 72.13 & 96.25 & 0.75 \\
\hline CNPN & 32 & 77.9 & 2.78 \\
\hline
\end{tabular}

The interactions between both types of CS chains and TPP as evidenced by the FTIR spectra prove nanoparticles formation. CS displays a broad band between $3500 \mathrm{~cm}^{-1}$ and approximately $3000 \mathrm{~cm}^{-1}$ corresponding to the combined stretching modes of the $\mathrm{O}-\mathrm{H}$ and N$\mathrm{H}$ bonds. In the CNP spectrum this band is broader due to an enhancement in hydrogen bonding and to the additional $-\mathrm{OH}$ groups introduced by TPP, and the intensities of the amide band $\left(\mathrm{CONH}_{2}\right)$ at $1658 \mathrm{~cm}^{-1}$ and amine $\left(\mathrm{NH}_{2}\right)$ band at $1562 \mathrm{~cm}^{-1}$ diminish. Two new peaks appear at $1637 \mathrm{~cm}^{-1}$ and at $1543 \mathrm{~cm}^{-1}$ upon treatment with TPP. The occurrence of the peaks at $\sim 1200 \mathrm{~cm}^{-1}(\mathrm{P}=\mathrm{O}$ stretching) and $887 \mathrm{~cm}^{-1}-891 \mathrm{~cm}^{-1}$ for P-O bending, after TPP addition, clearly evidence the crosslinking. FTIR spectra of CNPL/CNPX/

CNPN exhibit no significant differences compared to CNP. This result can be correlated to the very small amount of plant extract/nisin in the nanocomposites compared to CS. The release profiles for the bioactive agents from nanoparticles were evaluated over seven days and are presented in Figure 4. In all cases a burst was observed over the initial 4 hours, which may be due to the release of the $\mathrm{L} / \mathrm{X} / \mathrm{N}$ associated with the surface of the nanoparticles. After the initial burst, when the nanochitosan matrix started to degrade, all composites showed sustained release of the encapsulated species over seven days. The difference observed between $L$ and $\mathrm{X}$ profiles may be due to the difference in lipophylicity between L (more lipophilic) and $\mathrm{X}$. While displaying the same type of kinetics profile, CNPN released the highest percent over one week. Release times longer than seven days were not considered practical for applications in the food industry.

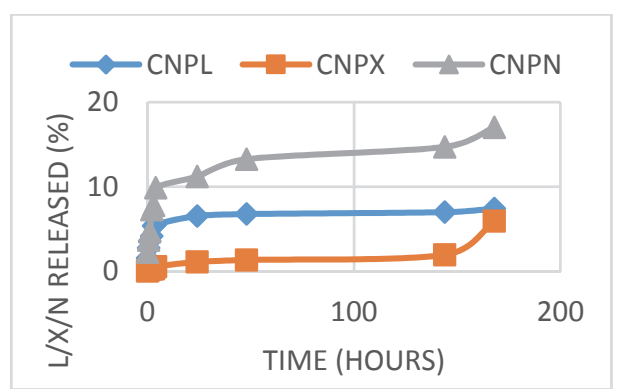

Figure 4. Release of $\mathrm{L} / \mathrm{X} / \mathrm{N}$ from nanochitosan matrices.

The antimicrobial activity of the nanoparticulate materials prepared was tested against a Gram-positive bacterium (Staphylococcus aureus) and two Gram negative (Pseudomonas aeruginosa and Escherichia coli), species involved in food spoilage. A higher activity was exhibited by CS and CNP samples against $S$. aureus compared to that against $P$. aeruginosa and E. coli (Table 3) The same behavior was displayed by the CNPL/CNPX/CNPN composites (with one exception) and was previously found in nanoparticulate chitosan encapsulating copper/silver ions [17]. The enhancement afforded by the nanosize was a decrease in MIC against both Gram negative bacteria by a factor of 2, and by $25 \%$ against S.aureus. Although the exact mechanism of action is not fully understood and several other factors may contribute to the antibacterial effect, Helander et al. [18] demonstrated that chitosan was able to bind and weaken the barrier function of the outer membrane of Gram-negative bacteria causing extensive cell surface alterations and possibly causing the loss of the barrier function. 
Table 3. Minimum inhibitory concentrations $(\mathrm{MIC}=\mu \mathrm{g} / \mathrm{ml})$ against $E$. coli, . aeruginosa, and S. aureus

\begin{tabular}{|c|c|c|c|c|c|c|c|}
\hline Bacterium & CS & CNP & CNP+1\% L & CNPL & CNPX & CNP+1\%N & CNPN \\
\hline E. coli & 1000 & 500 & 500 & 250 & 250 & & 62.5 \\
\hline P. aeruginosa & 1000 & 500 & 500 & 187 & 187 & 250 & 100 \\
\hline S. aureus & 500 & 375 & 187 & 125 & 187 & 125 & 125 \\
\hline
\end{tabular}

Zheng and Zhu [19] speculated that the thickness of the peptidoglycan layer can play an important role in providing a rigid structure which can act as a barrier against chitosan interactions. Raafat et al [20] related the effect of chitosan against Gram positive bacteria to its binding to teichoic acids, coupled with a potential extraction of membrane lipids (predominantly lipoteichoic acid) which result in a sequence of events, ultimately leading to leakage of the cell content and bacterial death and confirming the higher activity against Gram positive microbes. These explain the action of chitosan against both Gram positive and Gram-negative bacteria.

The nanocomposites are stronger antibacterial agents compared to CNP in spite of the very low active agent content. The activity of nanochitosan against $S$. aureus tripled upon addition of $\mathrm{L} / \mathrm{N}$, respectively and doubled for CNPX compared to CNP. More puzzling is the fact that synergistic effects were observed in the nanocomposites against the Gram-negative species although $\mathrm{L} / \mathrm{X} / \mathrm{N}$ are not active against them. To better evaluate the impact of the encapsulation of plant extract/nisin on the antibacterial activity, a set of experiments was conducted using L/N added to the solutions of CNP, respectively. As expected, concentrations of $1 \% \mathrm{~L}$ added to solutions of CNP did not impact MIC values against the Gram-negative bacteria but decreased MIC against $S$. aureus (1.33 times). Nisin additions (1\%) to CNP solutions resulted in higher impact on MIC values. While a decrease in MIC against the Grampositive $S$. aureus (by a factor of 3 compared to CNP and by a factor of 20 compared to nisin alone) was justified by the documented activity of nisin against Gram positive species, the synergy between CNP and added nisin reducing MIC against $P$. aeruginosa was not expected. The mechanism of action of nisin involves the disruption of cell membrane function due to its interaction with phospholipids and the formation of pores in the bacterial cell. When combined with chitosan, which is able to bind and weaken the barrier function of the outer membrane of Gram-negative bacteria causing extensive cell surface alterations and loss of the barrier function [18], nisin was able to penetrate and reach the plasma membrane where it interacted with the phospholipid and formed pores. This allowed for extensive cytoplasm leakage much faster than it does in Gram positive bacteria which have large amounts of peptidoglycan.

The synergistic effects afforded by the encapsulation of antibacterial species in nanochitosan matrices are stronger compared to those afforded by the simple addition of $\mathrm{L} / \mathrm{N}$ to $\mathrm{CNP}$ solutions if we take into account that the concentration of the added $\mathrm{L} / \mathrm{N}$ was two orders of magnitude higher than that of $\mathrm{L} / \mathrm{N}$ present in the nanocomposites. A comparison between the antibacterial effects of the nanoparticles discussed herein is presented in Figure 5.

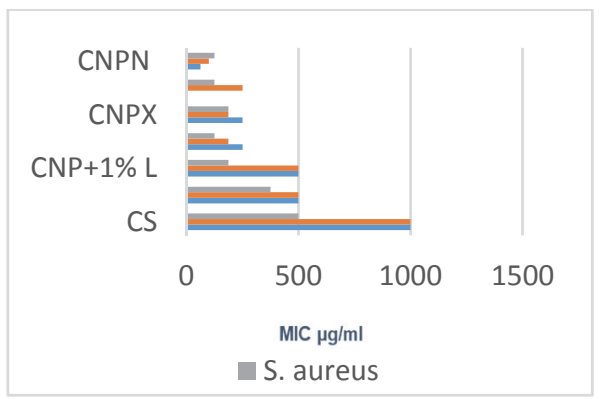

Figure 5. Comparison of MIC values of different samples against $S$. aureus $(+), P$. aeruginosa (-) and E. coli (-)

\section{Conclusion}

To the best of our knowledge, the present work is the first describing the successful encapsulation of hop components and nisin, 
respectively, in nanochitosan matrices. The nanoparticles obtained were characterized and the kinetics of the release of the active agent from nanochitosan was studied. The encapsulation resulted in synergistic interactions between chitosan and hop extracts/nisin and increased the antimicrobial effect of $\mathrm{L} / \mathrm{X} / \mathrm{N}$ against a broad spectrum of species. An added benefit of delivering hop components in nanochitosan matrices is the possibility to mask their strong bitter taste. This is important for food applications where hops are used as preservatives. Encapsulation also recommends the nanoparticles reported herein for applications to the skin (as treatment or in cosmetic formulations) since it avoids the danger of contact dermatitis reported in hop pickers and affords sustained release of nisin from mixtures containing fats.

\section{References}

1. T.J. Aspden, J.D. Mason, and N.S. Jones, J. Pharm. Sci. 86 (1997) 509.

2. K.A. Janes, P. Calvo, and M.J. Alonso, Adv. Drug Delivery Rev. 47 (2001a) 83.

3. S. Sagoo, R. Board, and S. Roller, Food Microbiol. 19 (2002) 175.

4. H.K. No, S.P. Meyers, W. Prinyawiwatkul, and Z. Xu, J. Food Sci. 72 (2007) R87.

5. J. Delves-Broughton, Food Australia, 57 (2005) 525.

6. M.D. Leonida and I. Kumar, Bionanomaterials for skin regeneration (Springer-Nature, Heidelberg/

New York/London, 2016) pp. 91-101.

7. P. Natarajan, S. Reddy, I. Andrei, V.B.R. Ambati, M.D. Leonida, and G.J. Haas, Phytomedicine, 15, 2008, 194.

8. M.G. Malowicki and T.H. Shellhammer, J. Agric. Food Chem. 53 (2005) 4434.

9. M. Teuber and A.F. Schmalreck, Arch. Mikrobiol. 94 (1973) 159.

10. P.C. Allen, Avian Res. 51 (2007) 21.

11. A. Hartkorn, F. Hoffmann, H. Ajamieh, S. Vogel, J. Heilmann, A.L. Gerbes, A.M. Vollmar, and S. Zahler, J. Nat. Prod. 72 (2009) 1741.

12. L. Qi, Z. Xi, X. Jiang, C. Hu, and X. Zou, Carbohydrate Res., 339 (2004) 2693.

13. P. Calvo, C. Remunan-Lopez, J.L. VillaJato, and M.J. Alonso, Pharm. Res., 14 (1997) 1431.
14. S.A. Wazed, S. Rajendran, and M. Joshi, Carbohydrate Polym., 83 (2011) 438.

15. S. D'Souza, Adv. Pharm. 2014, Article ID 304757, doi: 10.1155/2014/304757.

16. Clinical and Laboratory Standards Institute, Methods for Dilution Antimicrobial Susceptibility Tests for Bacteria That Grow Aerobically, $10^{\text {th }}$ ed., CLSI document M07A10, 2015.

17. M.D. Leonida, S. Banjade, T. Vo, G. Anderle, G.J. Haas, and N. Philips, Int. J. Nano\&Biomaterials, 3 (2011) 316.

18. I.M. Helander, E.L. Nurmiaho-Lassila, R. Ahvenainen, J. Rhoades, and S. Roller, Int. J. Food Microbiol. 71 (2001) 235.

19. L.Y. Zheng and J.F. Zhu, Carbohydr. Polym. 54 (2003) 527.

20. D. Raafat, K. von Bargen, A. Haas, and H.G. Sahl, Appl. Environ. Microbiol., 74 (2008) 3764.

21. N. Garg, J.O. Trent, T-S Wang, C. Garcia de Gonzalo, S. Walker, and W.A. van der Donk, J Antibiot (Tokyo), 67 (2014) 133.doi: 10.1038/ja.2013.112. 